\title{
Localization of Random Walks in One-Dimensional Random Environments
}

\author{
A. O. Golosov \\ Chair of Probability Theory, Department of Mechanics and Mathematics, Moscow State University, \\ SU-117234 Moscow, USSR
}

\begin{abstract}
We consider a random walk on the one-dimensional semi-lattice $\mathbb{Z}_{+}$ $=\{0,1,2, \ldots\}$. We prove that the moving particle walks mainly in a finite neighbourhood of a point depending only on time and a realization of the random environment. The size of this neighbourhood is estimated. The limit parameters of the walks are also determined.
\end{abstract}

\section{Introduction. Formulation of the Problem and Results}

Let us consider a sequence $\mathscr{A}=\left\{(q(x), r(x), p(x)) ; x \in \mathbb{Z}_{+}=\{0,1,2, \ldots\}\right\}$ of random three-dimensional vectors whose components are non-negative, and $q(0)=0, q(x)$ $+r(x)+p(x)=1$ for any $x \in \mathbb{Z}_{+}$. We shall call such a sequence a random environment. A random process $\left(x(t): t \in \mathbb{Z}_{+}\right)$will be called a random walk in the random environment $\mathscr{A}$ if the conditional distribution of $\left(x(t): t \in \mathbb{Z}_{+}\right)$under the condition that $\mathscr{A}$ is fixed is the distribution of the Markov chain whose phase space is $\mathbb{Z}_{+}$, initial state is 0 , and probabilities of transitions from $x$ to $x-1, x, x+1$ are $q(x)$, $r(x), p(x)$, respectively; $x \in \mathbb{Z}_{+}$. We shall denote by $P(\cdot \mid \mathscr{A})$ probabilities of events depending on random walks if a realization of the random environment $\mathscr{A}$ is fixed. Probabilities of events calculated without the assumption that the random environment $\mathscr{A}$ is fixed (including events connected with any properties of the random environment) will be denoted by $P(\cdot)$.

We assume that the random vectors $(q(x), r(x), p(x))$ are mutually independent for different $x \in \mathbb{Z}_{+},(q(x), p(x))$ are identically distributed for $x \geqq 1$, and $r(x)$ are identically distributed for $x \in \mathbb{Z}_{+}$. Moreover, we assume that the sequences of random variables $\left(r(x): x \in \mathbb{Z}_{+}\right)$and $(q(x) / p(x): x \geqq 1)$ are independent, $E \ln (q(x) / p(x))=0, \quad E(\ln (q(x) / p(x)))^{2}=\sigma^{2} \in(0,+\infty), \quad E(1-r(x))^{-1}<+\infty$, $P\{r(x)>0\}>0$. Sinai [1] proved that for random walks in similar random environments with the phase space $\mathbb{Z}=\{\ldots,-1,0,1,2, \ldots\}$ one can construct random variables $m(t)\left(t \in \mathbb{Z}_{+}\right)$depending only on $t$ and a random environment such that $x(t)-m(t)=o\left(\ln ^{2} t\right)$ (in probability) as $t \rightarrow+\infty$, and there exists the limit distribution of $m(t) \cdot(\ln t)^{-2}$ as $t \rightarrow+\infty$ which coincides with that of $x(t) \cdot(\ln t)^{-2}$. An 
analogous statement can be proved in our case too (see [2]). The results of the papers [1] and [2] mean that the conditional distribution of $x(t)$ under the condition that an environment $\mathscr{A}$ is fixed is concentrated in a small (with respect to $\ln ^{2} t, t \rightarrow+\infty$ ) neighbourhood of $m(t)$ (depending on a realization of the environment). We shall strengthen these results under the assumptions formulated above in the following way.

Theorem. There exists a random process $m(t)\left(t \in \mathbb{Z}_{+}\right)$depending only on $\mathscr{A}$, for which

(i) for any $y \in \mathbb{R}$ there exists

$$
\lim _{t \rightarrow+\infty} P\{x(t)-m(t) \leqq y\}=F(y)
$$

where $F$ is a distribution function;

(ii) the finite-dimensional distributions of the processes $(x(t+u)-m(t): u \in \mathbb{Z})$ converge as $t \rightarrow+\infty$ to ones of a stationary random process $(y(u): u \in \mathbb{Z})$ (for definiteness we put $x(t) \equiv 0$ for $t<0)$; the environment is not fixed;

(iii) if $r(x) \equiv$ const $<1$ and $\ln (q(x) / p(x))=\sigma \cdot \xi(x)$, where $E \xi(x)=0, E \xi^{2}(x)=1$, $\sigma>0, F_{\sigma}$ is the corresponding distribution function from assertion (i) of the theorem, then

$$
\lim _{\sigma \rightarrow 0} F_{\sigma}\left(x / \sigma^{2}\right)=G(x),
$$

where $G$ is a distribution function.

The assertions (i) and (ii) of the theorem mean that the moving particle walks mainly in a finite neighbourhood of $m(t)$ if $t$ is very large; assertion (iii) allows us to estimate the size of this neighbourhood: its value is as large as $\left(E(\ln (q(x) / p(x)))^{2}\right)^{-1}$.

The limit distributions $F$ and $G$ and the random process $(y(u): u \in \mathbb{Z})$ will be constructed in Sect. 2. The process $y$ is a random walk in a random environment with the phase space $\mathbb{Z}$ and non-independent non-stationary with respect to space translation transition probabilities, $F$ is the one-dimensional distribution of $y(u)$, the distribution $G$ is a functional of two independent Bessel processes.

\section{Main Constructions}

Let us denote $\eta(z)=\ln (q(z) / p(z)), s(z)=\sum_{0<i \leqq z} \eta(i), \quad z=1,2,3, \ldots ; s(0)=0 ; s(x)$ $=(x-z) s(z+1)+(z+1-x) s(z)$ if $z \leqq x<z+1$. Random variables $\eta(z)(z=1,2, \ldots)$ are mutually independent and identically distributed. Let us introduce random variables $\sigma(0)=0, \sigma(k)=\min \{z: z>\sigma(k-1), s(z)<s(\sigma(k-1))\}$ which are strict descending ladder epochs for $s(z), \alpha(k)=\max \{s(z)-s(\sigma(k)): \sigma(k) \leqq z \leqq \sigma(k+1)\}$, $k \in \mathbb{Z}_{+} ; n(y)=\min \{k: \alpha(k) \geqq y\}, y>0 ; v(t)=n\left(\ln t+(\ln t)^{1 / 2}\right), m(t)=\sigma(v(t))$. The point $m(t)$ is the nearest to 0 local minimum of the function $s(x)$ with properties: $s(x)$ $>s(m(t))$ for $0 \leqq x<m(t)$ and there exists $a_{t}>m(t)$ such that $s(m(t)) \leqq s(x) \leqq s\left(a_{t}\right)$ for $m(t) \leqq x \leqq a_{t}, s\left(a_{t}\right)-s(m(t)) \geqq \ln t+(\ln t)^{1 / 2}$.

Let us denote $\tau(z)=\min \{t: x(t)=z\}$. 
Lemma 1. For any $\varepsilon>0$

$$
\lim _{t \rightarrow+\infty} P\{\tau(m(t)) \leqq \varepsilon t\}=1
$$

Proof. See the appendix.

Let us describe the random environment in the neighbourhood of $m(t)$. Here and later we shall describe it in terms of the function $s(x)$ : it is clear that the realization of the environment $\mathscr{A}$ can be reconstructed if one knows the realization of $s(x)$ and the realization of the random variables $r(z)\left(z \in \mathbb{Z}_{+}\right)$which do not depend on the random function $s(x)$.

Let us consider a measurable space $(X, \mathscr{B})$ which is the union of the measurable spaces $\left(\mathbb{R}^{n}, \mathscr{B}^{n}\right), n=1,2, \ldots$, where $\mathscr{B}^{n}$ is the $\sigma$-field of Borel subsets of $\mathbb{R}^{n}$. Let us introduce the operation of sticking $A: X \times X \rightarrow X$; if $v=\left(x_{0}, \ldots, x_{n}\right) \in \mathbb{R}^{n+1}$, $w=\left(y_{0}, \ldots, y_{m}\right) \in \mathbb{R}^{m+1}$, then $v \Lambda w=\left(x_{0}, \ldots, x_{n-1}, \quad x_{n}+y_{0}, \quad x_{n}+y_{1}, \ldots, x_{n}+y_{m}\right)$ $\in \mathbb{R}^{n+m+1}$. Also we introduce the operation of turning: if $v=\left(x_{0}, \ldots, x_{n}\right) \in \mathbb{R}^{n+1}$, then the turned vector $v$ is $v^{\prime}=\left(x_{0}+\left(x_{n}-x_{n-i}\right): i=0, \ldots, n\right) \in \mathbb{R}^{n+1}$. Let us put $S_{k}=(s(\sigma(k)+i)-s(\sigma(k)): 0 \leqq i \leqq \sigma(k+1)-\sigma(k)) \in X$.

Lemma 2. $S_{k}\left(k \in \mathbb{Z}_{+}\right)$are independent identically distributed random elements of $(X, \mathscr{B})$. The distribution of $S_{k}$ coincides with the distribution of part of the realization of $s(z)\left(z \in \mathbb{Z}_{+}\right)$from $z=0$ to the first negative component.

Proof. The statement of Lemma 2 is a simple corollary of the strict Markov property of $s(z)\left(z \in \mathbb{Z}_{+}\right)$.

Let us denote $N(A)=\min \{z: z \geqq 1, s(z) \in A\}$. In view of Lemma 2 and the construction of $m(t)$, it is easy to demonstrate that the distribution of $\theta_{t}(k)$ $=s(m(t)+k)-s(m(t))\left(k \in \mathbb{Z}_{+}\right)$and the conditional distribution of $s(k)\left(k \in \mathbb{Z}_{+}\right)$under the condition that $N\left(\left[\ln t+(\ln t)^{1 / 2},+\infty\right)\right)<N((-\infty, 0))$ are the same.

The investigation of the distribution of $\theta_{t}(k)\left(k \in \mathbb{Z}_{+}\right)$is based on the following

Lemma 3. Let $x(t)\left(t \in \mathbb{Z}_{+}\right)$be a homogeneous Markov chain whose phase space is $Y$, an initial state is $x_{0} \in A \subseteq Y$, and transition probabilities for $x \in Y$ are $p(x, d y)$. Assume that $Y \backslash A=B \cup C, \quad B \cap C=\emptyset, \quad P\left\{\tau_{B}<\tau_{C}\right\}>0, \quad$ where $\tau_{B}=\min \{t \geqq 0: x(t) \in B\}$, $\tau_{C}=\min \{t \geqq 0: x(t) \in C\}$. Then the conditional distribution of $\left(x(0), \ldots, x\left(\tau_{B}\right)\right)$ under the condition that $\tau_{B}<\tau_{C}$ and the distribution of $\left(y(0), \ldots, y\left(\tau_{B}\right)\right)$ are the same, where $y(t)$ is a homogeneous Markov chain whose phase space is $Y \backslash C$, an initial state is $x_{0}$, and transition probabilities for $x \in A$ are $\bar{p}(x, d y)=p(x, d y) \cdot P_{y}\left\{\tau_{B}<\tau_{C}\right\} \cdot \chi_{Y \backslash C}(y)$ $\cdot\left(P_{x}\left\{\tau_{B}<\tau_{C}\right\}\right)^{-1}$; here $P_{x}(\cdot)$ denotes the distribution of the initial Markov chain whose initial state is $x$.

One can easily prove this lemma by means of simple calculations.

Lemma 4. Finite-dimensional probability distributions of $\theta_{t}(z)\left(z \in \mathbb{Z}_{+}\right)$converge to ones of $\theta(z)\left(z \in \mathbb{Z}_{+}\right)$as $t \rightarrow+\infty$, where $\theta(z)$ is a homogeneous Markov chain whose phase space is $[0,+\infty)$, the initial state is 0 , and transition probabilities for $x \in[0,+\infty)$ are $p(x, d y)=d H_{x}(y) \cdot f(y) \cdot(f(x))^{-1} \cdot \chi_{\{y \geqq 0\}}(y)$. Here $H_{x}(y)=H(y-x)$, $H(x)$ is the distribution function of $\ln (q(x) / p(x)), f(x)=\lim _{n \rightarrow+\infty} n \cdot P\{N([n,+\infty))$ $<N((-\infty,-x))\}$. Moreover, the distribution of $\left(\theta_{t}(0), \ldots, \theta_{t}(N([n,+\infty)))\right)$ is $a b$ - 
solutely continuous with respect to the distribution of $(\theta(0), \ldots, \theta(N([n,+\infty))))$, and $\lim _{t \rightarrow+\infty} P\left\{\left|\psi_{t}-1\right|>\varepsilon\right\}=0$, where $\psi_{t}$ is the corresponding Radon-Nikodym derivative, $P\{\cdot\}$ is the distribution of $\theta(z)\left(z \in \mathbb{Z}_{+}\right)$, and $\varepsilon$ is any positive number.

Proof. See the appendix.

Let us consider a sequence $\bar{S}_{1}, \bar{S}_{2}, \ldots, \bar{S}_{n}, \ldots$ of independent identically distributed random elements of $X$ whose distribution coincides with one of $(s(0), \ldots, s(N([0,+\infty))))$. Let us construct a random sequence $\bar{\theta}(z)\left(z \in \mathbb{Z}_{+}\right)$in the following way: $\bar{\theta}=\left(\bar{S}_{1}\right)^{\prime} \Lambda\left(\bar{S}_{2}\right)^{\prime} \Lambda\left(\bar{S}_{3}\right)^{\prime} \Lambda \ldots\left[\right.$ i.e. $\bar{\theta}=\lim _{k \rightarrow+\infty} \bar{\theta}_{k}$, where $\bar{\theta}_{k}=\bar{\theta}_{k-1} \Lambda\left(\left(\bar{S}_{k}\right)^{\prime}\right)$, $\bar{\theta}_{1}=\left(\bar{S}_{1}\right)^{\prime}$, and the limit is taken in the sense of the convergence of coordinates].

Lemma 5. The distributions of the random functions $\bar{\theta}$ and $\theta$ coincide.

Proof. See the appendix.

Before describing an asymptotic behaviour of the random environment to the left of $m(t)$ [asymptotic properties of the random environment to the right of $m(t)$ are described in Lemmas 4 and 5] we define infinitely deep well. By definition it is a random function $\theta(z)(z \in \mathbb{Z})$ with the following properties:

(i) $\theta(z) \geqq 0(z \in \mathbb{Z}), \theta(0)=0$;

(ii) $(\theta(z): z \geqq 0)$ and $(\theta(z): z \leqq 0)$ are independent random functions;

(iii) $(\theta(z): z \geqq 0)$ is the homogeneous Markov chain defined in Lemma 4;

(iv) $(\theta(0), \theta(-1), \theta(-2), \ldots)$ is a homogeneous Markov chain with the phase space $[0,+\infty)$, initial state 0 and transition probabilities $\bar{p}(x, d y)=d \bar{H}_{x}(y) \cdot \bar{f}(y)$ $\cdot(\bar{f}(x))^{-1} \cdot \chi_{(0,+\infty)}(y)$, where $\bar{H}_{x}(y)=\bar{H}(y-x), \bar{H}(x)$ is the distribution function of $(-\ln (q(z) / p(z))), \quad \bar{f}(x)=\lim _{n \rightarrow+\infty} n \cdot P\{N((-\infty,-n])<N([x,+\infty))\} \quad$ as $\quad x>0 ; \quad \bar{f}(0)$ $=\bar{f}(+0)$.

Lemma 6. Let $S_{k}\left(k \in \mathbb{Z}_{+}\right)$be independent identically distributed random elements of $X$ whose distribution coincides with one of $(s(0), s(1), \ldots, s(N((-\infty, 0))))$. Put $R_{k}=\left(0, x_{n-1}-x_{n}, \quad x_{n-2}-x_{n}, \ldots, x_{1}-x_{n},-x_{n}\right) \quad$ if $\quad S_{k}=\left(0, x_{1}, \ldots, x_{n}\right), \quad$ and $\theta^{*}=R_{0} \Lambda R_{1} \Lambda \ldots$, then the distributions of $\theta^{*}$ and $\left(\theta(-k): k \in \mathbb{Z}_{+}\right)$coincide.

Proof of lemma is analogous to that of Lemma 5.

Lemmas 5 and 6 give that $\lim _{|z| \rightarrow+\infty} \theta(z)=+\infty$ (this explains the term "infinitely deep well"). Using these lemmas and the properties of ladder epochs and ladder heights (see [3, pp. 395 and 575]), one can easily check that $\lim _{|z| \rightarrow+\infty} \theta(z) \cdot|z|^{-1 / 2+\varepsilon}$ $=+\infty$ for any $\varepsilon>0$ (with probability 1). It follows that the function $g(z)$ $=(\exp (-\theta(z))+\exp (-\theta(z-1))) \cdot\left(1-r^{\prime}(z)\right)^{-1} \cdot \Xi^{-1}(z \in \mathbb{Z})$ is the invariant distribution of the one-dimensional random walk in the environment $\mathscr{A}^{\prime}=\left(\left(q^{\prime}(z), r^{\prime}(z)\right.\right.$, $\left.\left.p^{\prime}(z)\right): z \in \mathbb{Z}\right)$, where $r^{\prime}(z)$ are independent identically distributed random variables not depending on $(\theta(z): z \in \mathbb{Z})$, whose distribution coincides with that of $r(z)\left(z \in \mathbb{Z}_{+}\right)$ for the initial random environment; using the equalities $q^{\prime}(z)+r^{\prime}(z)+p^{\prime}(z)=1$, $\ln \left(q^{\prime}(z) / p^{\prime}(z)\right)=\theta(z)-\theta(z-1)$, one can reconstruct $p^{\prime}(z)$ and $q^{\prime}(z)(z \in \mathbb{Z})$, if $(\theta(z): z \in \mathbb{Z})$ and $\left(r^{\prime}(z): z \in \mathbb{Z}\right)$ are known. 
This property of the random walk in the typical (with probability 1) realization of the random infinitely deep well implies that this is an ergodic Markov chain, and the transition probabilities converge to the invariant probabilities as $t \rightarrow+\infty$. Let $(y(u): u \in \mathbb{Z})$ be a random process such that the conditional distribution of $(y(u): u \in \mathbb{Z})$ under the condition that the realization of the random environment $\mathscr{A}^{\prime}$ is fixed is the distribution of the stationary ergodic Markov chain which is the random walk in this realization of the environment $\mathscr{A}^{\prime}$. The random process $y(u)$ is the limit process of the theorem.

Let us describe now the random environment to the left of $m(t)$. To obtain this environment we must take the random elements of $X: S_{0}, S_{1}, \ldots$ (see Lemma 6) until the first element $S_{k}$ such that $\max \left\{x_{i}: i=0, \ldots, \ell\right\} \geqq \ln t+(\ln t)^{1 / 2}$, where $S_{k}=\left(x_{0}, \ldots, x_{\ell}\right)$. The environment to the left of $m(t)$ is described by means of the function $R_{0} \Lambda R_{1} \Lambda \ldots \Lambda R_{k-1}$, where the correspondence between $S_{i}$ and $R_{i}$ is given in Lemma 6.

\section{Proof of the Theorem}

To prove (ii) of the theorem it is sufficient to check that

$$
P\left\{x(t+u+i)-m(t)=k_{i}: 0 \leqq i \leqq n\right\} \rightarrow P\left\{y(u+i)=k_{i}: 0 \leqq i \leqq n\right\}
$$

as $t \rightarrow+\infty$ for any $u \in \mathbb{Z}, n \geqq 0$ and $\mathbf{k}=\left(k_{0}, \ldots, k_{n}\right) \in \mathbb{Z}^{n+1}$. We shall demonstrate that the expectation of the random variable $\zeta_{t}(u, \mathbf{k})=P\left\{x(t+u+i)-m(t)=k_{i}: 0\right.$ $\leqq i \leqq n \mid \mathscr{A}\}$ converges to that of the random variable $\zeta(\mathbf{k})=P\left\{y(u+i)=k_{i}: 0\right.$ $\left.\leqq i \leqq n \mid \mathscr{A}^{\prime}\right\}$.

Denote $a(t)=\min \left\{z>m(t): s(z)-s(m(t)) \geqq \ln t+(\ln t)^{1 / 2}\right\}$. Let us consider a new random environment $\mathscr{A}_{1}(t)$ : if $0 \leqq z \leqq a(t)$, then the transition probabilities $q(z)$, $r(z), p(z)$ are the former ones, but if $z=a(t)+1$, then put $q(z)=1-r(z), p(z)=0$. In other words we place a reflecting barrier at $a(t)+1$. One can easily check that $S_{1}(t)$ $=(s(m(t)-z)-s(m(t)): 0 \leqq z \leqq m(t))$ and $S_{2}(t)=(s(z)-s(m(t)): m(t) \leqq z \leqq a(t))$ are independent elements of $X$.

Let us estimate $P\{\tau(a(t)+1)<3 t \mid \mathscr{A}\}$.

Lemma 7. For any $z \geqq 1$

$$
P\{\tau(z)<N \mid \mathscr{A}\} \leqq N \cdot \exp (-\max \{s(z-1)-s(i): 0 \leqq i<z\}) .
$$

Proof. Let $j \in \mathbb{Z}_{+}, 0 \leqq j<z$. Denote by $\alpha$ the number of steps from $j+1$ to the left during $[0, \tau(z))$, i.e. $\alpha=\#\left\{t: t \in \mathbb{Z}_{+}, 0 \leqq t<\tau(z), x(t)=j+1, x(t+1)=j\right\}$. Put $k(\mathscr{A})$ $=P\{\tau(z)<\tau(j) \mid x(0)=j+1 ; \mathscr{A}\}$. It is easy to demonstrate that the estimate $k(\mathscr{A})$ $\leqq \exp (s(j)-s(z-1))$ obtained by Sinai [1] for the case when $r(z) \equiv 0$ is also true in our situation. The inequalities $\tau(z) \geqq \alpha$ and

$$
P\{\alpha=\ell \mid \mathscr{A}\}=k(\mathscr{A}) \cdot(1-k(\mathscr{A}))^{\ell} \leqq k(\mathscr{A})
$$

give that $P\{\tau(z)<N \mid \mathscr{A}\} \leqq P\{\alpha<N \mid \mathscr{A}\} \leqq N \cdot k(\mathscr{A}) \leqq N \cdot \exp (s(j)-s(z-1))$. Therefore $P\{\tau(z)<N \mid \mathscr{A}\} \leqq N \cdot \exp (\min \{s(j)-s(z-1): 0 \leqq j<z\})$. Q.E.D.

Using this lemma we can estimate

$$
\begin{aligned}
P\{\tau(a(t)+1)<3 t \mid \mathscr{A}\} & \leqq 3 t \cdot \exp (-(s(a(t))-s(m(t)))) \leqq 3 t \cdot \exp \left(-\ln t-(\ln t)^{1 / 2}\right) \\
& =3 \exp \left(-(\ln t)^{1 / 2}\right) \rightarrow 0 \text { as } t \rightarrow+\infty .
\end{aligned}
$$


Denote $\zeta_{t}^{\prime}(u, \mathbf{k})=P\left\{x(t+u+i)-m(t)=k_{i}: 0 \leqq i \leqq n \mid \mathscr{A}_{1}(t)\right\}$, where $\mathbf{k}=\left(k_{0}, \ldots, k_{n}\right)$, $P\left(\cdot \mid \mathscr{A}_{1}(t)\right)$ is the distribution of the random walk in the new random environment $\mathscr{A}_{1}(t)$. From the estimate

$$
P\{\tau(a(t)+1)<3 t \mid \mathscr{A}\} \leqq 3 \exp \left(-(\ln t)^{1 / 2}\right),
$$

it follows that

$$
\left|\zeta_{t}^{\prime}(u, \mathbf{k})-\zeta_{t}(u, \mathbf{k})\right| \leqq 3 \exp \left(-(\ln t)^{1 / 2}\right)
$$

for any $u \leqq t(t>n)$.

Let us consider the random walk $z(u)(u \geqq 0)$ in the infinitely deep well [i.e. in the environment $\mathscr{A}^{\prime}=\left(\{\theta(z)\},\left\{r^{\prime}(z)\right\}\right)$ described in Sect. 2] such that $z(0)=0$. Denote

$$
\kappa(u, \mathbf{k})=P\left\{z(u+i)=k_{i}: 0 \leqq i \leqq n \mid \mathscr{A}^{\prime}\right\} .
$$

Let us construct a new random environment $\mathscr{A}^{\prime}(t)$ starting from the infinitely deep well: place reflecting barriers in $b(t)+1$, where

$$
b(t)=\min \left\{z: z \geqq 0, \theta(z) \geqq \ln t+(\ln t)^{1 / 2}\right\},
$$

and in $(-d(t))$, which can be found in the following way: let $S_{k}=\left(x_{0}, \ldots, x_{\ell}\right)$ be the first element in the sequence $\left(S_{i}: i \in \mathbb{Z}_{+}\right)$(see the formulation of Lemma 6) such that

Then

$$
\max \left\{x_{i}-x_{0}: 0 \leqq i \leqq \ell\right\} \geqq \ln t+(\ln t)^{1 / 2} .
$$

$$
R_{0} \Lambda R_{1} \Lambda \ldots \Lambda R_{k-1}=\left(y_{0}, \ldots, y_{d(t)}\right) \in \mathbb{R}^{d(t)+1} \cong X .
$$

One can easily check that

$$
\Theta_{1}(t)=(\theta(-z): 0 \leqq z \leqq d(t))
$$

and

$$
\Theta_{2}(t)=(\theta(z): 0 \leqq z \leqq b(t))
$$

are independent elements of $X$, and moreover the distributions of $\Theta_{1}(t)$ and $S_{1}(t)$ (for the environment $\mathscr{A}_{1}(t)$ ) coincide, the distribution of $S_{2}(t)$ is absolutely continuous with respect to one of $\Theta_{2}(t)$ (see Lemma 4), and the corresponding Radon-Nikodym derivative $\psi_{t}$ converges to 1 (in probability) as $t \rightarrow+\infty$.

Denote $z^{\prime}(u)$ the position of the randomly moving particle at the moment of time $u$ in the environment $\mathscr{A}^{\prime}(t)$; the initial position of the particle is 0 . Put $\kappa_{t}^{\prime}(u, \mathbf{k})$ $=P\left\{z^{\prime}(u+i)=k_{i}: 0 \leqq i \leqq n \mid \mathscr{A}^{\prime}(t)\right\}$, and denote $\left(g_{t}^{\prime}(z): z \in \mathbb{Z}\right)$ the invariant distribution of this finite Markov chain.

Let $\varepsilon>0$ be fixed. From the ergodicity of the process $y(u)$ (when the environment is fixed), it follows that the one-dimensional distribution of $z(u)$ converges to the invariant distribution $g=(g(z): z \in \mathbb{Z})$, where $g$ is a function of the environment. Consequently there exists $u_{0}(\varepsilon)$ such that $E\|\kappa(u)-g\|_{1}<\varepsilon$ for $u \geqq u_{0}(\varepsilon)$, where $\kappa(u)=(\kappa(u, \mathbf{k}): \mathbf{k} \in \mathbb{Z}),\|\cdot\|_{1}$ is the norm in $\ell_{1}(\mathbb{Z})$. It is easy to demonstrate that for any large enough $t E\left\|\kappa\left(u_{0}(\varepsilon)\right)-\kappa_{t}^{\prime}\left(u_{0}(\varepsilon)\right)\right\|_{1}<\varepsilon$, where $\kappa_{t}^{\prime}(u)$ $=\left(\kappa_{t}^{\prime}(u, \mathbf{k}): \mathbf{k} \in \mathbb{Z}\right)$. Therefore

$$
E\left\|\kappa_{t}^{\prime}\left(u_{0}(\varepsilon)\right)-g\right\|_{1}<2 \varepsilon
$$


for this $t$. One can check that the inequality $E\left\|g-g_{t}^{\prime}\right\|_{1}<\varepsilon$ holds for all sufficiently large $t$; from this inequality it follows that

$$
E\left\|\kappa_{t}^{\prime}\left(u_{0}(\varepsilon)\right)-g_{t}^{\prime}\right\|_{1}<3 \varepsilon,
$$

if $t$ is large enough. But $\left\|\kappa_{t}^{\prime}(u)-g_{t}^{\prime}\right\|_{1} \leqq\left\|\kappa_{t}^{\prime}\left(u_{0}(\varepsilon)\right)-g_{t}^{\prime}\right\|_{1}$ for $u \geqq u_{0}(\varepsilon)$ because $g_{t}^{\prime}$ is the invariant distribution for the corresponding Markov chain. Therefore $E\left\|\kappa_{t}^{\prime}(u)-g_{t}^{\prime}\right\|_{1}<3 \varepsilon$ for $u \geqq u_{0}(\varepsilon)$ and sufficiently large $t$. This gives $E\left\|\kappa_{t}^{\prime}(u)-g\right\|_{1}$ $<4 \varepsilon$, and

$$
E\left|\kappa_{t}^{\prime}(u, \mathbf{k})-\zeta(\mathbf{k})\right|<4 \varepsilon
$$

if $u \geqq u_{0}(\varepsilon)$ and $t$ is large enough, and

$$
\left|E \kappa_{t}^{\prime}(u, \mathbf{k})-E \zeta(\mathbf{k})\right|<4 \varepsilon
$$

for these $u$ and $t$. We have

$$
\zeta_{t}^{\prime}(u, \mathbf{k})=\sum_{\ell=0}^{+\infty} \zeta_{t}^{\prime}(\ell ; u, \mathbf{k}) \cdot P\left\{\tau(m(t))=\ell \mid \mathscr{A}_{1}(t)\right\}
$$

where

$$
\zeta_{t}^{\prime}(\ell ; u, \mathbf{k})=P\left\{x(t+u+i)=k_{i}: 0 \leqq i \leqq n \mid \mathscr{A}_{1}(t) ; \tau(m(t))=\ell\right\} .
$$

The correspondence between the distributions of the random environments $\mathscr{A}^{\prime}(t)$ and $\mathscr{A}_{1}(t)$ mentioned above gives that

$$
\left|E \zeta_{t}^{\prime}(\ell ; u, \mathbf{k})-E \kappa_{t}^{\prime}(t+u-\ell ; \mathbf{k})\right|<\varepsilon
$$

for $0 \leqq \ell \leqq u+t$ and sufficiently large $t$. Using this estimation and Lemma 1 we obtain

$$
\left|E \zeta_{t}^{\prime}(u, \mathbf{k})-E \zeta(\mathbf{k})\right|<6 \varepsilon,
$$

if $t$ is large enough and $u \geqq-t / 2$. From the inequalities (3.2) and (3.3) it follows that

$$
\left|E \zeta_{t}(u, \mathbf{k})-E \zeta(\mathbf{k})\right|<6 \varepsilon+3 \exp \left(-(\ln t)^{1 / 2}\right)<7 \varepsilon
$$

for sufficiently large $t$ and $u \in[-t / 2, t]$. Therefore $E \zeta_{t}(u, \mathbf{k}) \rightarrow E \zeta(\mathbf{k})$ as $t \rightarrow+\infty$ uniformly on $u \in[-t / 2, t]$, and (3.1) holds. The assertions (i) and (ii) of the theorem are proven.

Denote $g_{\sigma}(z)=\frac{1}{2}(\exp (-\sigma \theta(z))+\exp (-\sigma \theta(z-1))) \cdot \Xi_{\sigma}^{-1}$, the invariant distribution of the random walk in the infinitely deep well $\mathscr{A}_{\sigma}^{\prime}=(\{\sigma \theta(z): z \in \mathbb{Z}\}$, $\left.\left\{r^{\prime}(z): z \in \mathbb{Z}\right\}\right)$, where $\Xi_{\sigma}^{-1}$ is a normalizing factor, and put $F_{\sigma}(x)=\sum_{z \leqq x} g_{\sigma}(z)$. Let $(\bar{w}(s): s \geqq 0)$ and $(\bar{w}(-s): s \geqq 0)$ be independent identically distributed random processes whose distribution is one of the radial part of the three-dimen-

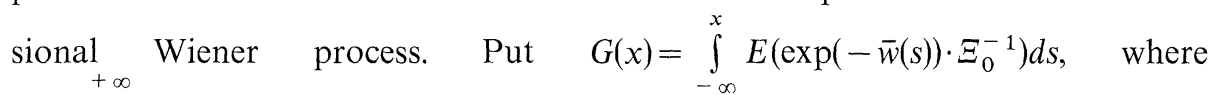
$\Xi_{0}=\int_{-\infty}^{\infty} \exp (-\bar{w}(s)) d s$ is a normalizing factor.

In order to complete the proof of the theorem it is sufficient to prove the following 
Assertion. For any $x \in \mathbb{R}$

$$
F_{\sigma}\left(x / \sigma^{2}\right) \rightarrow G(x) \quad \text { as } \quad \sigma \rightarrow 0 .
$$

Proof. Let us consider a random function $f_{\sigma}(x)=\exp (-\sigma \theta(x))$ for $x \in \mathbb{Z}$, and the value of $f_{\sigma}(x)$ for $x \notin \mathbb{Z}$ we define by means of the linear interpolation. We have $\Xi_{\sigma}=\int_{-\infty}^{+\infty} f_{\sigma}(x) d x$. Put $h_{\sigma}(x)=f_{\sigma}(x) \cdot \Xi_{\sigma}^{-1}$. One can easily check that the weak limits of the distributions $d F_{\sigma}\left(x / \sigma^{2}\right)$ and $E \sigma^{-2} h_{\sigma}\left(x / \sigma^{2}\right) d x$ coincide.

Lemma 8. The common probability distribution of

converges to one of

$$
\left(\left(f_{\sigma}\left(x / \sigma^{2}\right): x \in \mathbb{R}\right), \sigma^{2} \Xi_{\sigma}\right)
$$

$$
\left((\exp (-\bar{w}(x)): x \in \mathbb{R}), \Xi_{0}\right)
$$

as $\sigma \rightarrow 0$; here we claim the convergence of distributions in $C(\mathbb{R}) \times \mathbb{R}$, where $C(\mathbb{R})$ is provided with the topology of uniform convergence on compact subsets of $\mathbb{R}$.

Proof. See the appendix.

Form Lemma 8 it follows that the distributions of the continuous random functions $\sigma^{-2} h_{\sigma}\left(x / \sigma^{2}\right)$ converge to one of $h_{0}(x)=\exp (-\bar{w}(x)) \cdot \Xi_{0}^{-1}$ as $\sigma \rightarrow 0$. For any $x \in \mathbb{R}$ we have $\sigma^{-2} h_{\sigma}\left(x / \sigma^{2}\right) \geqq 0, h_{0}(x) \geqq 0$, and one-dimensional distributions of the random functions $\sigma^{-2} h_{\sigma}\left(x / \sigma^{2}\right)$ converge to ones of $h_{0}(x)$. Therefore

$$
\liminf _{\sigma \rightarrow 0} E \sigma^{-2} h_{\sigma}\left(x / \sigma^{2}\right) \geqq E h_{0}(x) \quad(x \in \mathbb{R}) .
$$

Using Fatou's lemma we have

$$
\begin{aligned}
G(b)-G(a) & =\int_{a}^{b} E h_{0}(x) d x \leqq \int_{a}^{b}\left(\liminf _{\sigma \rightarrow 0} E \sigma^{-2} h_{\sigma}\left(x / \sigma^{2}\right)\right) d x \\
& \leqq \liminf _{\sigma \rightarrow 0} \int_{a}^{b} E \sigma^{-2} h_{\sigma}\left(x / \sigma^{2}\right) d x \\
& =\liminf _{\sigma \rightarrow 0}\left(\Phi_{\sigma}\left(b / \sigma^{2}\right)-\Phi_{\sigma}\left(a / \sigma^{2}\right)\right),
\end{aligned}
$$

where $-\infty \leqq a<b \leqq+\infty$, and $\Phi_{\sigma}(x)=E \int_{-\infty}^{x} h_{\sigma}(s) d s$. We have $G(b) \leqq \liminf _{\sigma \rightarrow 0} \Phi_{\sigma}\left(b / \sigma^{2}\right)$ for $a=-\infty$, and $1-G(a) \leqq \liminf _{\sigma \rightarrow 0}\left(1-\Phi_{\sigma}\left(a / \sigma^{2}\right)\right)=1-\limsup _{\sigma \rightarrow 0} \Phi_{\sigma}\left(a / \sigma^{2}\right)$ for $b=$
$+\infty$.

Therefore there exists $\lim _{\sigma \rightarrow 0} \Phi_{\sigma}\left(x / \sigma^{2}\right)=G(x)$ for any $x \in \mathbb{R}$, and $\lim _{\sigma \rightarrow 0} F_{\sigma}\left(x / \sigma^{2}\right)=G(x)$, because the limits of $F_{\sigma}\left(x / \sigma^{2}\right)$ and $\Phi_{\sigma}\left(x / \sigma^{2}\right)$ coincide (this fact was mentioned above). Q.E.D.

The assertion (iii) of the theorem is proven.

\section{Appendix. Proofs of Lemmas}

Proof of Lemma 1. Let us prove the inequality

$$
\begin{aligned}
E\left(\tau(z) \mid s(x), x \in \mathbb{Z}_{+}\right) \leqq & \left(E(1-r(z))^{-1}\right) \\
& \cdot z^{2} \exp (\max \{s(v)-s(u): 0 \leqq u \leqq v<z\}) .
\end{aligned}
$$


Denote $\alpha(z)=\max \{s(v)-s(u): 0 \leqq u \leqq v<z\}, \quad \beta_{i}=\#\{t: t<\tau(z), \quad x(t)=i\} \quad(i=0,1$, $\ldots, z-1), \pi_{i}=P$ the moving particle arrive at $z$ earlier then returned to $i \mid \mathscr{A}$; $x(0)=i\}$. The equality

$$
\pi_{i}=p(i)\left(\sum_{k=i}^{z-1} \exp (s(k)-s(i))\right)^{-1}
$$

is analogous to the similar formula for the probability of non-returning of the moving particle to the initial state obtained by Sinai [1], and can be checked with the same methods.

From the obvious equality

$$
P\left\{\beta_{i}=\ell \mid \mathscr{A}\right\}=\left(1-\pi_{i}\right)^{\ell-1} \cdot \pi_{i} \quad(\ell=1,2, \ldots),
$$

it follows that $E\left(\beta_{i} \mid \mathscr{A}\right)=\pi_{i}^{-1}$. We have

$$
\begin{aligned}
\pi_{i}^{-1}= & (1-r(i))^{-1}(p(i)+q(i)) \cdot(p(i))^{-1} \cdot \sum_{k=1}^{z-1} \exp (s(k)-s(i)) \\
= & (1-r(i))^{-1}\left(1+(\exp (s(i)-s(i-1)))\left(1-\delta_{0, i}\right)\right) \\
& \cdot \sum_{k=i}^{z-1} \exp (s(k)-s(i)) .
\end{aligned}
$$

Therefore $E\left(\beta_{i} \mid \mathscr{A}\right) \leqq(1-r(i))^{-1} \cdot\left(2-\delta_{0, i}\right)(z-i) \exp (\alpha(z))$, and

$$
E\left(\beta_{i} \mid s(x), x \in \mathbb{Z}_{+}\right) \leqq\left(E(1-r(x))^{-1}\right)\left(2-\delta_{0, i}\right)(z-i) \exp (\alpha(z)) .
$$

Using (A.2) and the equality $\tau(z)=\beta_{0}+\ldots+\beta_{z-1}$, we obtain (A.1).

Let us estimate $\varphi_{t}=\max \{s(v)-s(u): 0 \leqq u \leqq v<m(t)\}$. It is easy to demonstrate that the functionals $\mu(s)=\min \{u: \exists v>u$ such that $s(v)-s(u)=1, s(u) \leqq s(t) \leqq s(v)$ as $u \leqq t \leqq v\}$ and $\varphi(s)=\sup \{s(v)-s(u): 0 \leqq u \leqq v \leqq \mu(s)\}$ are determined and continuous in $C([0,+\infty)), \mathscr{W}$-almost surely, where $\mathscr{W}$ is the Wiener measure in $C([0,+\infty))$, $s \in C([0,+\infty))$. The distribution of continuous random function $s(t \cdot u)$ $\cdot\left(t \sigma^{2}\right)^{-1 / 2}(u \in[0,+\infty))$ converges to the Wiener measure $\mathscr{W}$ in $C([0,+\infty))$. Therefore the distributions of $\varphi_{t} \cdot\left(\ln t+(\ln t)^{1 / 2}\right)^{-1}$ and $m(t) \cdot\left(\ln t+(\ln t)^{1 / 2}\right)^{-2} \cdot \sigma^{2}$ converge to ones of the functionals $\varphi(w)$ and $\mu(w)$, respectively, where $w$ is the Wiener process (see Theorem 5.1 in $[4$, p. 30]). It is clear that the limits of the distributions of $\varphi_{t} \cdot(\ln t)^{-1}$ and $m(t) \cdot(\ln t)^{-2} \cdot \sigma^{2}$ are the same. One can calculate the limit distribution of $\varphi_{t}$ considering the case when $\ln (q(z) / p(z))= \pm 1$ with probabilities $1 / 2$. The explicit expression of this distribution is not important for us, we shall use only the fact that it is absolutely continuous and concentrated in the interval $[0,1]$. Using (A.1) we can estimate

$$
\begin{aligned}
& P\left\{\tau(m(t))>\varepsilon t \mid s(x): x \in \mathbb{Z}_{+}\right\} \leqq(\varepsilon t)^{-1} \\
& \quad \cdot E\left(\tau(m(t)) \mid s(x): x \in \mathbb{Z}_{+}\right) \leqq \text {const } \cdot(\varepsilon t)^{-1}(m(t))^{2} \cdot \exp \left(\varphi_{t}\right)
\end{aligned}
$$

[we used the fact that $m(t)$ depends only on $s(x)$ but not the whole random environment $\mathscr{A}]$. We have $P\left\{B\left(\varepsilon_{1}, t\right)\right\}>1-\varepsilon_{1}$ for any $\varepsilon_{1}>0$, suitable $\delta=\delta\left(\varepsilon_{1}\right)$, and sufficiently large $t$, where $B\left(\varepsilon_{1}, t\right)=\left\{\mathscr{A}: m(t)<\delta^{-1} \cdot(\ln t)^{2}, \quad \varphi_{t}<(1-\delta) \ln t\right\}$. 
Therefore

$$
\begin{aligned}
& P\left\{\tau(m(t))>\varepsilon t \mid s(x): x \in \mathbb{Z}_{+}\right\} \leqq \text {const } \cdot(\varepsilon t)^{-1} \delta^{-2} \\
& \cdot(\ln t)^{4} \cdot t^{1-\delta} \rightarrow 0(t \rightarrow+\infty)
\end{aligned}
$$

if $s \in B\left(\varepsilon_{1}, t\right)$, and $P\{\tau(m(t))>\varepsilon t\} \rightarrow 0$ as $t \rightarrow+\infty$ because $\varepsilon_{1}$ is any positive number. Q.E.D.

Proof of Lemma 4. From Lemma 3 it follows that it is sufficient to show that

$$
\lim _{n \rightarrow+\infty} n \cdot P\{A(n, x)\}=f(x),
$$

where $f(x)=-E s(N((-\infty,-x)))$, and

$$
E(s(N([n,+\infty)))-n \mid A(n, x))=o(n)
$$

as $n \rightarrow+\infty$, where $A(n, x)=\{N([n,+\infty))<N((-\infty,-x))\}$.

Let us derive (A.4) from (A.3). Denote $N=\min \{N([n,+\infty)), N((-\infty,-x))\}$. The random function $s(z)\left(z \in \mathbb{Z}_{+}\right)$is a martingale. Therefore

$$
0=E s(N)=P\{A(n, x)\} E(s(N) \mid A(n, x))+P\left\{A^{\prime}(n, x)\right\} E\left(s(N) \mid A^{\prime}(n, x)\right),
$$

where $A^{\prime}(n, x)=\{N([n,+\infty))>N((-\infty,-x))\}$. From the equality (A.3), it follows that $P\{A(n, x)\} \sim f(x) / n, P\left\{A^{\prime}(n, x)\right\}=1-f(x) / n+o\left(n^{-1}\right), E\left(s(N) \mid A^{\prime}(n, x)\right)$ $=\operatorname{ES}(N((-\infty,-x)))+o(1)(n \rightarrow+\infty)$. Therefore

$$
\begin{aligned}
& E(S(N([n,+\infty))) \mid A(n, x))-n \\
& \quad=-\left(f(x) / n+o\left(n^{-1}\right)\right)^{-1}\left(1-f(x) / n+o\left(n^{-1}\right)\right)(-f(x)+o(1))-n \\
& \quad=o(n)(n \rightarrow+\infty) .
\end{aligned}
$$

Let us prove (A.3). Considering the simplest random environment for which $\ln (q(z) / p(z))= \pm 1$ with probabilities $1 / 2$, we can demonstrate that the distribution of the random variable

$$
\gamma(t)=-s(m(t)) / \ln t
$$

converges as $t \rightarrow+\infty$ to the exponential distribution with the expectation 1 [because $-w(\mu(w))$ is a continuous functional of the Wiener process $w$ a.s.]. The distribution of $\gamma(t)$ can also be written in terms of $N([n,+\infty))$ and $N((-\infty,-x))$. Let us consider a new random variable $m_{x}(t)$ : put $\sigma_{x}(0)=0, \sigma_{x}(k)=\min \{z: z$ $\left.>\sigma_{x}(k-1), s(z)<s\left(\sigma_{x}(k-1)\right)-x\right\}, \alpha_{x}(k)=\max \left\{s(z)-s\left(\sigma_{x}(k)\right): \sigma_{x}(k) \leqq z \leqq \sigma_{x}(k+1)\right\}$, $n_{x}(y)=\min \left\{k: \alpha_{x}(k) \geqq y\right\}, v_{x}(t)=n_{x}(\ln t), m_{x}(t)=\sigma_{x}\left(v_{x}(t)\right)$. Using the weak convergence of the continuous random function $s\left(x \cdot \ell^{2} \cdot \sigma^{-2}\right) \cdot \ell^{-1}$ to the Wiener process as $\ell \rightarrow+\infty$ and properties of the functionals $\varphi_{t}$ (see proof of Lemma 1), one can check that $m_{x}(t)-m(t)=o\left(\ln ^{2} t\right)$ (in probability) as $t \rightarrow+\infty$. Therefore the limit distributions of random variables $\gamma(t)$ and $\gamma_{x}(t)=-s\left(m_{x}(t)\right) / \ln t$ coincide. We have

$$
-s\left(m_{x}(t)\right)=\sum_{i=0}^{v_{x}(t)-1} \zeta_{x}(t, i),
$$

where random variables $\zeta_{x}(t, i)=s\left(\sigma_{x}(i)\right)-s\left(\sigma_{x}(i+1)\right)$ are independent and identically distributed $\left(0 \leqq i<v_{x}(t)\right)$. The distribution of $\zeta_{x}(t, i)$ coincides with the conditional distribution of $(-s(N((-\infty,-x))))$ under the condition that $N((-\infty$, 
$-x))<N([\ln t,+\infty))$. The distribution of $v_{x}(t)$ can be also calculated:

$$
P\left\{v_{x}(t)=i\right\}=\varrho_{t}^{i} \cdot\left(1-\varrho_{t}\right),
$$

where $i \in \mathbb{Z}_{+}$, and $\varrho_{t}=P\left\{N((-\infty,-x))<N([\ln t,+\infty))\right.$. Therefore $E\left(-s\left(m_{x}(t)\right)\right)$ $\sim f(x) \cdot\left(1-\varrho_{t}\right)^{-1}$ as $t \rightarrow+\infty$, and the distribution of

$$
\left(-s\left(m_{x}(t)\right)\right) \cdot\left(1-\varrho_{t}\right) / f(x)
$$

converges to the exponential one with the expectation 1. Comparing the normalizing factors in (A.5) and (A.6), we obtain

$$
\left(1-\varrho_{t}\right) \cdot \ln t \rightarrow f(x), \quad t \rightarrow+\infty,
$$

and therefore the equality (A.3) holds. Q.E.D.

Proof of Lemma 5. Denote by $\pi$, $\mu$, and $v$ probability measures on $\left(\mathbb{R}^{\mathbb{Z}_{+}}, \mathscr{B}^{\mathbb{Z}_{+}}\right)$ corresponding to the random processes $\left(s(z): z \in \mathbb{Z}_{+}\right),\left(\bar{\theta}(z): z \in \mathbb{Z}_{+}\right)$and $\left(\theta(z): z \in \mathbb{Z}_{+}\right)$, respectively. The distribution of the vector consisting of the first $n+1$ coordinates of $\mathbf{x}=\left(x_{0}, x_{1}, \ldots\right) \in \mathbb{R}^{\mathbb{Z}_{+}}$we shall denote by $d \pi\left(x_{0}, \ldots, x_{n}\right), d \mu\left(x_{0}, \ldots, x_{n}\right)$, $d v\left(x_{0}, \ldots, x_{n}\right)$. Note that $d v\left(x_{0}, \ldots, x_{n}\right)=d \pi\left(x_{0}, \ldots, x_{n}\right) \cdot \chi_{\left\{x_{i} \geqq 0: 0 \leqq i \leqq n\right\}}(x) \cdot f\left(x_{n}\right) / f(0)$. To prove Lemma 5 it is sufficient to demonstrate the equality $d \mu\left(x_{0}, \ldots, x_{n}\right)$ $=d v\left(x_{0}, \ldots, x_{n}\right)$ for any $n \geqq 0$, i.e. to check that

$$
\int h d \mu\left(x_{0}, \ldots, x_{n}\right)=\int h d v\left(x_{0}, \ldots, x_{n}\right)
$$

for any measurable map $h: \mathbb{R}^{n+1} \rightarrow \mathbb{R}$ such that

$$
\sup \left\{|h(\mathbf{x})|: x \in \mathbb{R}^{n+1}\right\} \leqq \text { const } .
$$

Denote by $\gamma$ the map of $X^{\mathbb{N}}$ into $\mathbb{R}^{\mathbb{Z}_{+}}$defined before the formulation of Lemma $5, \gamma:\left(\bar{S}_{1}, \bar{S}_{2}, \ldots\right) \mapsto\left(\bar{\theta}(z): z \in \mathbb{Z}_{+}\right)$. The vector $(\bar{\theta}(0), \ldots, \bar{\theta}(n))$ depends on not more than $n$ initial terms of $\left(\bar{S}_{1}, \bar{S}_{2}, \ldots\right)$ because $\left|\bar{S}_{i}\right| \geqq 1$ for any $i$ (a.s.), where by definition $|v|=n$ if $v \in \mathbb{R}^{n+1}$; it is clear that $|v \Lambda w|=|v|+|w|$. Let us consider subsets of $X^{\mathbb{N}}$

$$
A\left(\ell_{1}, \ldots, \ell_{k}\right)=\left\{\left(\bar{S}_{1}, \bar{S}_{2}, \ldots\right):\left|\bar{S}_{i}\right|=\ell_{i}, 1 \leqq i \leqq k\right\},
$$

where

$$
\ell_{1} \geqq 1, \ldots, \ell_{k} \geqq 1, \quad \ell_{1}+\ldots+\ell_{k-1}<n, \quad \ell_{1}+\ldots+\ell_{k-1}+\ell_{k} \geqq n .
$$

Using this notation we have

$$
\begin{array}{rl}
\int_{\mathbb{R}^{n+1}} & h d \mu\left(x_{0}, \ldots, x_{n}\right) \\
= & \int_{\mathbb{R}^{\mathbb{N}}} h(\mathbf{x}(\bar{\theta})) d \mu(\bar{\theta})=\int_{X^{\mathbb{N}}} h(\mathbf{x}(\gamma(\mathbf{v}))) d P(\mathbf{v}) \\
= & \sum_{\left(\ell_{1}, \ldots, \ell_{k}\right)} \int_{X^{\mathbb{N}}} h(x(\gamma(\mathbf{v}))) \cdot \chi_{A\left(\ell_{1}, \ldots, \ell_{k}\right)}(\mathbf{v}) d P(\mathbf{v}) \\
= & \sum_{\left(\ell_{1}, \ldots, \ell_{k}\right)} g\left(\ell_{1}, \ldots, \ell_{k}\right),
\end{array}
$$


where $\mathbf{x}\left(\alpha_{0}, \alpha_{1}, \alpha_{2}, \ldots\right)=\left(\alpha_{0}, \ldots, \alpha_{n}\right), P(\cdot)$ is the distribution on $\left(X^{\mathbb{N}}, \mathscr{B}^{\mathbb{N}}\right)$ corresponding to the random sequence $\left(\bar{S}_{1}, \bar{S}_{2}, \ldots\right)$. Denote

$$
\gamma_{k}:\left(\bar{S}_{1}, \ldots, \bar{S}_{k}\right) \mapsto\left(\bar{S}_{1}\right)^{\prime} \Lambda \ldots \Lambda\left(\bar{S}_{k}\right)^{\prime} \in \mathbb{R}^{\ell+1},
$$

where $\ell=\ell_{1}+\ldots+\ell_{k},\left|\bar{S}_{i}\right|=\ell_{i}, i=0, \ldots, n$. We have

$$
g\left(\ell_{1}, \ldots, \ell_{k}\right)=\int_{X^{\mathbb{N}}} h\left(\mathbf{x}^{\prime}\left(\gamma_{k}(\mathbf{v})\right)\right) \chi_{A\left(\ell_{1}, \ldots, \ell_{k}\right)}(\mathbf{v}) d P(\mathbf{v}),
$$

where $\mathbf{x}^{\prime}\left(\alpha_{0}, \ldots, \alpha_{\ell}\right)=\left(\alpha_{0}, \ldots, \alpha_{n}\right)$. Using the definition of $\bar{S}_{i}$ and the property of duality of sums of independent random variables (see [3, p. 377]), one can check that

$$
\begin{aligned}
& g\left(\ell_{1}, \ldots, \ell_{k}\right) \\
& =\int d \pi(\mathbf{y}) h\left(x^{\prime}(\mathbf{y})\right) \prod_{i=0}^{k-1} \chi_{\left\{y_{j}>y_{m_{2}+1}: m_{2}<j<m_{i+1}\right\}}(\mathbf{y}) \\
& \quad \cdot \chi_{\left\{0 \leqq y_{m_{1}} \leqq \ldots \leqq y_{m_{k}}\right\}}(\mathbf{y}),
\end{aligned}
$$

where $m_{0}=0, m_{i}=m_{i-1}+\ell, m_{k}=\ell, \mathbf{y}=\left(y_{0}, \ldots, y_{\ell}\right)$. Denote

$$
\begin{aligned}
& \varphi_{j}(u, v, w) \\
& =\int d \pi(\mathbf{y}) \chi_{\left\{y_{1}>y_{j}, \ldots, y_{j-1}>y_{j}\right\}}(\mathbf{y}) \cdot \chi_{\left\{y_{j} \geqq v-u\right\}}(\mathbf{y}) \\
& \quad \cdot \chi_{\left\{y_{J}<w-u\right\}}(\mathbf{y}) \cdot \chi_{\{u \geqq w \geqq v\}}(u, v, w),
\end{aligned}
$$

where $j \geqq 1, \mathbf{y}=\left(y_{0}, \ldots, y_{j}\right)$. We have

$$
\begin{aligned}
g\left(\ell_{1}, \ldots, \ell_{k}\right) & \\
= & \int d \pi\left(y_{0}, \ldots, y_{n}\right) h\left(y_{0}, \ldots, y_{n}\right) \\
& \cdot \prod_{i=0}^{k-2} \chi_{\left\{y_{j}>y_{m_{i+1}}: m_{t}<j<m_{t}+1\right\}}\left(y_{0}, \ldots, y_{n}\right) \\
& \cdot \chi_{\left\{0 \leqq y_{m_{1}} \leqq \ldots \leqq y_{m_{k-1}}\right\}}\left(y_{0}, \ldots, y_{n}\right) \\
& \cdot \varphi_{\ell-n}\left(y_{n}, y_{m_{k-1}}, \min \left\{y_{j}: m_{k-1}<j \leqq n\right\}\right) .
\end{aligned}
$$

Let us find

$$
\begin{aligned}
& \sum_{i=1}^{+\infty} \varphi_{i}(u, v, w) \\
& =\sum_{i=1}^{+\infty} P\{i \text { is a strict descending ladder epoch for } s(z), \\
& =\sum_{i=1}^{+\infty} \sum_{j=1}^{+\infty} P\left\{i \text { is the } j^{\text {th }} \text { strict descending ladder epoch for } s(z),\right. \\
& =\sum_{j=1}^{+\infty} P\left\{\text { the value of } s(\cdot) \text { in the } j^{\text {th }} \text { strict descending }(i) \in[v-u, w-u)\right\} \\
& =U((u-w, u-v]),
\end{aligned}
$$


where $U=\beta+\beta * \beta+\beta * \beta * \beta+\ldots, \beta$ is the distribution of the first strict ascending ladder height for $(-s(z))$.

By means of simple transformations we obtain

$$
\begin{aligned}
& \sum_{\left(\ell_{1}, \ldots, \ell_{k}\right)} g\left(\ell_{1}, \ldots, \ell_{k}\right) \\
& =\sum_{\left(\ell_{1}, \ldots, \ell_{k-1}\right)}\left(\sum_{i=1}^{+\infty} g\left(\ell_{1}, \ldots, \ell_{k-1}, n-m_{k-1}+i\right)+g\left(\ell_{1}, \ldots, \ell_{k-1}, n-m_{k-1}\right)\right) \\
& =\int d \pi\left(x_{0}, \ldots, x_{n}\right) h\left(x_{0}, \ldots, x_{n}\right) \\
& \quad \cdot \sum_{\left(\ell_{1}, \ldots, \ell_{k-1}\right)}\left(\prod_{i=0}^{k-2} \chi_{\left\{x_{j}>x_{m_{i+1}}: m_{i}<j<m_{i+1}\right\}}(\mathbf{x})\right. \\
& \quad \cdot \chi_{\left\{0 \leqq x_{m_{1}} \leqq \ldots \leqq x_{m_{k-1}}\right\}}(\mathbf{x}) U\left(\left(x_{n}-\min \left\{x_{j}: m_{k-1}<j \leqq n\right\}, x_{n}-x_{m_{k-1}}\right]\right) \\
& \quad+\chi_{\left\{x_{j}>x_{m_{1}}: m_{0}<j<m_{1}\right\}}(\mathbf{x}) \cdot \ldots \cdot \chi_{\left\{x_{j}>x_{n}: m_{k-1}<j<n\right\}}(\mathbf{x}) \\
& \left.\quad \cdot \chi_{\left\{0 \leqq x_{m_{1}} \leqq \ldots \leqq x_{m_{k-1}} \leqq x_{n}\right\}}(\mathbf{x})\right) \\
& =\int h\left(x_{0}, \ldots, x_{n}\right)\left(\chi_{[0,+\infty)}\left(x_{n}\right)+U\left(\left(0, x_{n}\right]\right)\right) \\
& \quad \cdot d \pi\left(x_{0}, \ldots, x_{n}\right),
\end{aligned}
$$

because for any vector $\mathbf{x}=\left(x_{0}, \ldots, x_{n}\right)$ we can define $\ell_{1}(\mathbf{x})=\min \left\{i: i>0, x_{j} \geqq x_{i}\right.$ as $j>i\}, \ell_{2}(\mathbf{x})=\min \left\{i: i>\ell_{1}(x), x_{j} \geqq x_{i}\right.$ as $\left.j>i\right\}, \ldots, \ell_{k(\mathbf{x})}=n$, the expression appearing in (A.7) under the symbol $\sum$ does not equal 0 only for the following vectors $\left(\ell_{1}, \ldots, \ell_{k-1}\right): \emptyset,\left(\ell_{1}(\mathbf{x})\right),\left(\ell_{1}(\mathbf{x}), \ell_{2}(\mathbf{x})\right), \ldots,\left(\ell_{1}(\mathbf{x}), \ldots, \ell_{k(\mathbf{x})-1}(\mathbf{x})\right)$, and this expression equals $U\left(\left(x_{n}-x_{\ell_{1}(\mathbf{x})}, x_{n}\right]\right), U\left(\left(x_{n}-x_{\ell_{2}(\mathbf{x})}, x_{n}-x_{\ell_{1}(\mathbf{x})}\right]\right), \ldots, U\left(\left(0, x_{n}-x_{\ell_{k(\mathbf{x})-1}}(\mathbf{x})\right]\right)$ $+\chi_{[0,+\infty)}\left(x_{n}\right)$, respectively. Finally we obtain

$$
d \mu\left(x_{0}, \ldots, x_{n}\right)=d \pi\left(x_{0}, \ldots, x_{n}\right) \cdot\left(\chi_{[0,+\infty)}\left(x_{n}\right)+U\left(\left(0, x_{n}\right]\right)\right) .
$$

It is sufficient now to show that $\chi_{[0,+\infty)}(x)+U((0, x])=f(x) / f(0)$. Using the fact that the functions $f(x) / f(0)$ and $\chi_{[0,+\infty)}(x)+U((0, x])$ are monotonous, and the equalities

$$
d v\left(x_{0}, \ldots, x_{n}\right)=d \pi\left(x_{0}, \ldots, x_{n}\right) \cdot \chi_{\left\{x_{i} \geqq 0: 0 \leqq i \leqq n\right\}}(\mathbf{x}) \cdot f\left(x_{n}\right) / f(0),
$$

and (A.8), one can easily check that these functions are solutions of the equation

$$
\int_{[0,+\infty)} g(y) d H_{x}(y)=g(x), x \in[0,+\infty)
$$

with the boundary condition $g(x)=0$ as $x<0$, where $H_{x}(y)=H(y-x), H$ is the distribution function of $\ln (q(z) / p(z))$. Equation (A.9) has only one asymptotically linear solution up to a multiplicative constant, and we now use the fact that $f(x)$ $\sim$ const $\cdot x$ as $x \rightarrow+\infty, U((0, x]) \sim$ const $\cdot x$ as $x \rightarrow+\infty$ (see [3, p. 347]). Lemma 5 is proven.

Proof of Lemma 8. Let us denote by $C(\mathbb{R})$ the space of real-valued continuous functions defined on $\mathbb{R}$. Let us supply $C(\mathbb{R})$ with the topology of uniform convergence on compact subsets of $\mathbb{R}$. Extend the function $\theta$ from $\mathbb{Z}$ to $\mathbb{R}$ by means of linear interpolation. We shall prove that the distributions in $C(\mathbb{R})$ corresponding to the random functions $\sigma \theta\left(x / \sigma^{2}\right)(x \in \mathbb{R})$ converge as $\sigma \rightarrow 0$ to the distribution corresponding to the process $\bar{w}(x)$. Denote by $\mu$ the distribution of random continuous function $w(x)$ such that $(w(x): x \geqq 0)$ and $(w(-x): x \geqq 0)$ are 
independent Wiener processes. Let us consider the map $\alpha: C(\mathbb{R}) \rightarrow C(\mathbb{R})$, $\alpha: \varphi \mapsto \alpha[\varphi]$ such that $(\alpha[\varphi](x): x \geqq 0)$ depends only on $(\varphi(x): x \geqq 0)$, $(\alpha[\varphi](-x): x \geqq 0)$ depends in the same manner on $(\varphi(-x): x \geqq 0)$. Now we shall describe the construction of $(\alpha[\varphi](x): x \geqq 0)$. Denote $\bar{\varphi}(x)=\max \{\varphi(y): 0 \leqq y \leqq x\}$, $\psi(x)=\bar{\varphi}(x)-\varphi(x)$; the open set $\{x: x \in(0,+\infty), \psi(x)>0\}$ is the union of intervals $I_{k}=I_{k}(\varphi)=\left(\beta_{k}(\varphi), \gamma_{k}(\varphi)\right), k \in \mathbb{Z}$, for $\mu$-a.s. $\varphi \in C(\mathbb{R})$. Put $\tilde{\psi}(x)=0$ if $\psi(x)=0, \tilde{\psi}(x)$ $=\psi\left(\gamma_{k}(\varphi)-\left(x-\beta_{k}(\varphi)\right)\right)$ if $x \in I_{k}(\varphi)$. Finally, put $\alpha[\varphi](x)=\bar{\varphi}(x)+\tilde{\psi}(x), x \geqq 0$. The map $\alpha$ is continuous in $\varphi \in C(\mathbb{R})$ such that the values of $\varphi$ in different local extrema of $\varphi$ are different. The $\mu$-measure of the set of such functions $\varphi$ equals 1 because the distributions of extremal values of $w(x)$ in any fixed interval are absolutely continuous. Therefore the map $\alpha$ is continuous almost everywhere in $C(\mathbb{R})$. From this fact it follows that distributions of continuous random functions $\left(\alpha\left[s_{\sigma}\right](x): x \in \mathbb{R}\right)$ converge as $\sigma \rightarrow 0$ to the distribution of $(\alpha[w](x): x \in \mathbb{R})$, where $s_{\sigma}(x)=\sigma s\left(x / \sigma^{2}\right)$. The distribution of $(\alpha[w](x): x \in \mathbb{R})$ coincides with that of the random function $\bar{w}(x)=2 \max \{w(y): y$ lies between 0 and $x\}-w(x)$, where $x \in \mathbb{R}$. To demonstrate this fact it is sufficient to consider again the simplest case when $\xi(z)= \pm 1$ with probabilities $1 / 2$ : in this case the distribution of $\bar{\varphi}(x)+\tilde{\psi}(x)$ coincides with that of $\bar{\varphi}(x)+\psi(x)=2 \bar{\varphi}(x)-\varphi(x)$. Now it remains to use Pitman's theorem (see [5]). From the independence of random processes $(\theta(x): x \geqq 0$ ) and $(\theta(x): x \leqq 0)$, it follows that it is sufficient to prove that the distributions in $C([0,+\infty))$ corresponding to $\left(\sigma \theta\left(x / \sigma^{2}\right): x \geqq 0\right)$ and $\left(\sigma \theta\left(-x / \sigma^{2}\right): x \geqq 0\right)$ converge as $\sigma \rightarrow 0$ to the distribution of $(\bar{w}(x): x \geqq 0)$. We shall check only the convergence of the distribution of the first of these two processes, the second case can be investigated analogously.

Let us consider a map $\alpha_{\sigma}$ defined on the set of continuous functions which are linear on the intervals $\left[\sigma^{2} \cdot k, \sigma^{2} \cdot(k+1)\right]\left(k \in \mathbb{Z}_{+}\right)$. If $I_{k}(\varphi)=\left(\beta_{k}(\varphi), \gamma_{k}(\varphi)\right)$ and $\gamma_{k}(\varphi) \in\left(\sigma^{2} \cdot n, \sigma^{2} \cdot(n+1)\right]$, then for $x \in\left[\left(\gamma_{k}(\varphi)-n \sigma^{2}\right)+\beta_{k}(\varphi),(n+1) \sigma^{2}\right]$, put $\alpha_{\sigma}[\varphi](x)$ $=\alpha[\varphi]\left(x-\left((n+1) \sigma^{2}-\gamma_{k}(\varphi)\right)\right)+\left(\alpha[\varphi]\left(\sigma^{2} \cdot(n+1)\right)-\alpha[\varphi]\left(\gamma_{k}(\varphi)\right)\right) ;$ for other $x \in[0,+\infty)$ put $\alpha_{\sigma}[\varphi](x)=\alpha[\varphi](x)$. If $\varphi$ does not belong to the set of functions described above, then we put $\alpha_{\sigma}[\varphi]=\alpha[\varphi]$. Let $A>0$ be fixed. We have

$$
\begin{aligned}
& \sup \left\{\left|\alpha_{\sigma}[\varphi](x)-\alpha[\varphi](x)\right|: x \in[0, A]\right\} \\
& \quad \leqq 2 \sup \left\{|\alpha[\varphi](y)-\alpha[\varphi](x)|:|x-y| \leqq \sigma^{2} ; x, y \in\left[0, A+\sigma^{2}\right]\right\} .
\end{aligned}
$$

for any function $\varphi \in C([0,+\infty))$ from the domain of definition of $\alpha$. Thus the distributions of $\alpha_{\sigma}\left[s_{\sigma}\right]$ converge as $\sigma \rightarrow 0$ to the distribution of $\alpha[w](x)$ and $\bar{w}(x)$ $(x \in[0,+\infty))$. The distributions of $\sigma \bar{\theta}\left(x / \sigma^{2}\right)$ and $\sigma \theta\left(x / \sigma^{2}\right)(x \in[0,+\infty))$ are the same (see Lemma 5), and $\alpha_{\sigma}\left[s_{\sigma}\right]=\sigma \bar{\theta}\left(x / \sigma^{2}\right)$. Therefore the distribution of $\left(\sigma \theta\left(x / \sigma^{2}\right): x \in[0,+\infty)\right)$ converges to one of $(\bar{w}(x): x \geqq 0)$ as $\sigma \rightarrow 0$. Using the inequality

$$
\begin{aligned}
& \max \left\{\left|\exp \left(-\sigma \theta\left(x / \sigma^{2}\right)\right)-f_{\sigma}\left(x / \sigma^{2}\right)\right|: 0 \leqq x \leqq A\right\} \\
& \quad \leqq \max \left\{\left|\exp \left(-\sigma \theta\left(x / \sigma^{2}\right)\right)-\exp \left(-\sigma \theta\left(y / \sigma^{2}\right)\right)\right|:|x-y| \leqq \sigma^{2} ; x, y \in\left[0, A+\sigma^{2}\right]\right\},
\end{aligned}
$$

(where $A>0$ is arbitrary) and convergence of the distribution of $\left(\sigma \theta\left(x / \sigma^{2}\right): x \geqq 0\right)$ to that of $(\bar{w}(x): x \geqq 0)$ as $\sigma \rightarrow 0$, one can easily prove that the distribution of $\left(f_{\sigma}\left(x / \sigma^{2}\right): x \geqq 0\right)$ converges to that of $(\exp (-\bar{w}(x)): x \geqq 0)$ as $\sigma \rightarrow 0$. The convergence of the distribution of $\left(f_{\sigma}\left(x / \sigma^{2}\right): x \leqq 0\right)$ to that of $(\exp (-\bar{w}(x)): x \leqq 0)$ can be proven analogously. Therefore $\left(f_{\sigma}\left(x / \sigma^{2}\right): x \in \mathbb{R}\right) \rightarrow(\exp (-\bar{w}(x)): x \in \mathbb{R})$ as $\sigma \rightarrow 0$ (in distri- 
bution). The independence of $\left(f_{\sigma}(x): x \leqq 0\right)$ and $\left(f_{\sigma}(x): x \geqq 0\right)$ and similar properties of $\bar{w}$ should be also used.

To complete the proof of Lemma 8 it is sufficient to to demonstrate that

$$
\int_{\mathbb{R} \backslash[-A, A]} f_{\sigma}\left(x / \sigma^{2}\right) d x=\int_{(-\infty,-A)} f_{\sigma}\left(x / \sigma^{2}\right) d x+\int_{(A,+\infty)} f_{\sigma}\left(x / \sigma^{2}\right) d x
$$

is infinitely small (in probability) as $A \rightarrow+\infty$ uniformly on $\sigma \in(0,1)$, because the functional

$$
\zeta:(\varphi(x): x \in \mathbb{R}) \mapsto\left((\varphi(x): x \in \mathbb{R}), \int_{(-A, A)} \varphi(x) d x\right)
$$

is continuous on $C(\mathbb{R})$. We shall check this assertion only for the second integral on the right side of (A.10), the first one can be estimated analogously. We have

$$
\int_{(A,+\infty)} f_{\sigma}\left(x / \sigma^{2}\right) d x \leqq \sigma^{2} \sum_{\sigma^{2} z>A-\sigma^{2}} f_{\sigma}(z)=\sigma^{2} \sum_{\sigma^{2} z>A-\sigma^{2}} \exp (-\sigma \theta(z)) \text {. }
$$

Denote $\bar{S}(z)=\max \{s(i): i \leqq z\}$. Using Lemma 5 and inequality

we obtain

$$
\sigma \bar{\theta}(z)=\alpha_{\sigma}\left[s_{\sigma}\right]\left(\sigma^{2} z\right) \geqq \sigma \bar{s}(z) \text {, }
$$

$$
\sum_{\sigma^{2} z>A-\sigma^{2}} \exp (-\sigma \bar{\theta}(z)) \leqq \sum_{\sigma^{2} z>A-\sigma^{2}} \exp (-\sigma \bar{S}(z)) .
$$

Let us consider random variables $\tau_{k}=\tau_{k}(\sigma, A)$ :

Denote

$$
\begin{gathered}
\tau_{0}=\min \left\{z: z \in \mathbb{Z}_{+}, \sigma^{2} z>A-\sigma^{2}, \bar{s}(z)>\bar{s}\left(A \sigma^{-2}-1\right)\right\}, \\
\tau_{k}=\min \left\{z: z \in \mathbb{Z}_{+}, z>\tau_{k-1}, \bar{s}(x)>\bar{s}\left(\tau_{k-1}\right)\right\}, \quad k=1,2, \ldots .
\end{gathered}
$$

We have

$$
\begin{gathered}
\zeta_{k}=\bar{s}\left(\tau_{k}\right)-\bar{s}\left(\tau_{k-1}\right), \quad B(\sigma, A)=\exp \left(-\sigma \bar{s}\left(A \sigma^{-2}-1\right)\right), \\
D(\sigma, A)=\left(\tau_{0}-A \sigma^{-2}+2\right) \cdot \sigma^{2}, \\
K(\sigma, A)=\sigma^{2} \sum_{n=0}^{+\infty}\left(\left(\tau_{n+1}-\tau_{n}\right) \exp \left(-\sigma \sum_{i=1}^{n} \zeta_{i}\right)\right) .
\end{gathered}
$$

$$
\sigma^{2} \sum_{\sigma^{2} z>A-\sigma^{2}} \exp (-\sigma \bar{s}(z)) \leqq B(\sigma, A) D(\sigma, A)+B(\sigma, A) K(\sigma, A)
$$

From the convergence of the distribution of the continuous random function $a^{-1 / 2} s(a x)(x \in[0,+\infty))$ to that of the Wiener process as $a \rightarrow+\infty$, it follows that there exist limit distributions as $A \sigma^{-2} \rightarrow+\infty$ of the random variables $\kappa(\sigma, A)$ $=\bar{s}\left(A / \sigma^{2}-1\right) \cdot\left(A / \sigma^{2}\right)^{-1 / 2}$ and $d(\sigma, A)=D(\sigma, A) A^{-1}$, and these limit distributions are concentrated on $[0,+\infty)$. Therefore, random variables $B(\sigma, A)$ $=\exp \left(-A^{1 / 2} \kappa(\sigma, A)\right)$ and $B(\sigma, A) D(\sigma, A)=\exp \left(-A^{1 / 2} \kappa(\sigma, A)\right) \cdot A \cdot d(\sigma, A)$ are infinitely small as $A \rightarrow+\infty$ uniformly on $\sigma \in(0,1)$. To finish the proof of Lemma 8 it remains only to check that $K(\sigma, A)$ is bounded (in probability) as $A \rightarrow+\infty$ uniformly on $\sigma \in(0,1)$. Random vectors $\left(\tau_{k+1}-\tau_{k}, \zeta_{k+1}\right)$ are independent and identically distributed $\left(k \in \mathbb{Z}_{+}\right)$, and the distribution of these vectors does not depend on $A$ and $\sigma$. We have

$$
\mu=E \zeta_{k}<+\infty, \quad P\left\{\tau_{k+1}-\tau_{k}>y\right\} \sim \text { const } \cdot y^{-1 / 2} \quad \text { as } \quad y \rightarrow+\infty
$$


(see [3, pp. 575 and 395]). Denote by $\varphi(\lambda)$ the logarithm of the Laplace transformation of the random variables $\tau_{k+1}-\tau_{k} ; \varphi(\lambda) \sim$ const $\cdot \lambda^{1 / 2}(\lambda \rightarrow 0)$ because (A.11). Let us estimate

$$
K_{1}(\sigma, A)=\sigma^{2} \sum_{n=0}^{+\infty}\left(\tau_{\left[\sigma^{-1}\right] \cdot(n+1)}-\tau_{\left[\sigma^{-1}\right] \cdot n}\right) \cdot \exp (-n \mu / 2) .
$$

We have

$$
P\left\{K(\sigma, A) \leqq K_{1}(\sigma, A)\right\} \geqq P\left\{\sum_{i=1}^{[\sigma-1] \cdot n} \zeta_{i} \geqq \mu n \sigma^{-1} / 2: n=1,2, \ldots\right\} \geqq 1-\varepsilon
$$

when $\sigma \in\left(0, \sigma_{0}\right)$, where $\varepsilon$ is any positive number and $\sigma_{0}=\sigma_{0}(\varepsilon)$. One can easily check that

$$
\begin{aligned}
E \exp \left(-\lambda K_{1}(\sigma, A)\right) & =\exp \left(\sum_{n=0}^{+\infty}\left[\sigma^{-1}\right] \cdot \varphi\left(\sigma^{2} \exp (-n \mu / 2) \cdot \lambda\right)\right) \\
& \geqq \exp \left(\text { const } \cdot \lambda^{1 / 2}\right) .
\end{aligned}
$$

Therefore random variables $K_{1}(\sigma, A)$ are bounded in probability as $A \rightarrow+\infty$ uniformly on $\sigma \in(0,1)$. Let $\varepsilon>0$ be fixed. The estimation

$$
P\{K(\sigma, A)>y\} \leqq \varepsilon+P\left\{K_{1}(\sigma, A)>y\right\} \leqq 2 \varepsilon
$$

holds when $y>y_{0}(\varepsilon), \sigma \in\left(0, \sigma_{0}\right)$; the estimation

$$
\begin{aligned}
K(\sigma, A) \leqq & \sum_{n=0}^{+\infty}\left(\tau_{k+1}\left(\sigma_{0}(\varepsilon), A\right)-\tau_{k}\left(\sigma_{0}(\varepsilon), A\right)\right) \\
& \cdot \exp \left(-\sigma_{0}(\varepsilon) \sum_{i=1}^{n} \zeta_{i}\right)=L_{\varepsilon}(A)
\end{aligned}
$$

holds when $\sigma \in\left[\sigma_{0}, 1\right)$, where the distribution of the random variable $L_{\varepsilon}(A)$ does not depend on $A$. Therefore

$$
P\{K(\sigma, A)>y\}<\varepsilon,
$$

as $y>y_{1}(\varepsilon)$ uniformly on $\sigma \in\left[\sigma_{0}, 1\right)$ and $A>0$. Finally, the random variables $K(\sigma, A)$ are bounded in probability uniformly on $\sigma \in(0,1)$ as $A \rightarrow+\infty$. Lemma 8 is proven.

Acknowledgements. The author expresses his cordial gratitude to Professor Ya. G. Sinai for the formulation of the problem and valuable discussions.

\section{References}

1. Sinai, Ya.G.: Limit behaviour of one-dimensional random walk in random environment. Theory Probab. Appl. 27, No. 2, 247-258 (1982) (in Russian)

2. Golosov, A.O.: Limit distributions for random walks in random environments. Dokl. Acad. Sci. USSR 271, 25-29 (1983) (in Russian)

3. Feller, W.: An introduction to probability theory and its applications, Vol. 2. New York, London, Sydney: Wiley 1966

4. Billingsley, P.: Convergence of probability measures. New York, London, Sydney, Toronto: Wiley 1968

5. Pitman, J.W.: One-dimensional Brownian motion and the three-dimensional Bessel process. Adv. Appl. Probab. 7, 511-526 (1975)

Communicated by Ya. G. Sinai

Received April 15, 1983 